\title{
"Snake": New Object of Hypothetical Venusian Fauna
}

\author{
Leonid V. Ksanfomality \\ Space Research Institute of the Russian Academy of Sciences, Moscow, Russia \\ Email: ksanf@rssi.ru
}

Received 6 November 2013; revised 5 December 2013; accepted 13 December 2013

Copyright (C) 2014 by author and Scientific Research Publishing Inc.

This work is licensed under the Creative Commons Attribution International License (CC BY). http://creativecommons.org/licenses/by/4.0/

(c) (i) Open Access

\begin{abstract}
On March 1 and 5, 1982, experiments in television photography instrumented by the landers VENERA-13 and 14 returned panoramas of the Venus surface at the landing site. Over the past 31 years, no similar missions have been sent to Venus. In connection with the renewed interest in what was occurring during the experiment and to the discovery of manifestations of possible life revealed on some of the pictures, the panoramas were re-examined. Analysis of treated once again VENERA-14 panoramic images revealed a "snake" object about $40 \mathrm{~cm}$ in size possessing apparent terramorphic features. The snake's body stands out with its honeycomb, spotty surface against the stone plates close by. The "snake" can be included into the list of the most significant findings of the hypothetical Venusian fauna. Apart from that, of interest is a "dove" object, although details of its structure cannot be discerned. The snake's body show slow movements, which is another evidence of the Venusian fauna's very slow style of activity, which appears to be associated with its energy constraints, and that is more likely, with the properties of its internal medium. The terramorphic features of the fauna, if they are confirmed, may point out outstandingly important and yet undiscovered general laws of the animated nature.
\end{abstract}

\section{Keywords}

Planet Venus' Fauna; Astrobiology; Space Vehicles Instruments; Planet's Surface

\section{Introduction. Some Data on the Venera Experiments}

On March 1 and 5, 1982, experiments in television photography instrumented by the landers Venera 13 and 14, yielded 33 panoramas of the Venus surface (or their fragments) at the landing site. The methodology of the television experiments on the surface of Venus and the date and list of the experimental data have been discussed in detail in [1]-[3] and are not repeated here. Some remarks on the critical features of the treatment used are given 
below. Over the past 31 years, no similar missions have been sent to Venus. In connection with the renewed interest in what was occurring during the experiment and to the discovery of manifestations of possible life revealed on some of the pictures, the panoramas were re-examined. A train of papers [2]-[4] published in 2012 presented some data on the hypothetical Venus fauna that survives under physical settings that are radically different from the Earth's. Along with unfamiliar forms, some of the found objects are closely reminiscent of the forms of some of Earth's living organisms. The phenomenon is called terramorphism. E.g., the term might be referred to as a "bear cub" [4], as found in a VENERA-9 panorama (Figure 1). The resolution in the panorama is 21 ', and for that reason, despite the bear cub's position, the identifiability of finer details is limited.

Still more pronounced terramorphism, or similarity to the Earth's fauna, can be found in the "snake" whose features are discussed in this paper. The phenomenon of terramorphism, which is observed on another planet and in totally different natural settings, in case it is confirmed, can note as yet unknown laws of living form development and can render prospects for further advances in the biosciences.

As noted in earlier published papers [2] [5] [6], during the years that passed since the time of TV experiments that employed the VENERA-9, -10 (1975) and VENERA-13, 14 (1982) landers, no similar experiments have been performed. Papers [2]-[6] addressed the findings of revision and novel treatment as well as the analysis of the VENERA-9 and VENERA-13 panoramic images. Going farther than the publications mentioned above, this paper offers the results of a new analysis of VENERA-14 lander panoramas, in which new interesting and unusual findings were revealed.

The object discussed in this paper drew researchers' attention before (in 1983) due to the uncommon geological features of the region [7].

The objects of hypothetical fauna that were found at both landing points (VENERA-13 and VENERA-14) are different (with a single exception), and the differences could be related, in a way, to the features of the landing sites. The coordinates of the VENERA- 14 landing site are $13^{\circ} \mathrm{S} / 310^{\circ} \mathrm{E}$, with a site altitude of $1.3 \mathrm{~km}$ above the $6051 \mathrm{~km}$ radius level and a temperature of $738 \mathrm{~K}$ and pressure of 9.47 MPa. A fragment of the area map (in the northern part of Panina Patera) is shown in Figure 2.

It is essential to give some data on the planet surface properties at the VENERA-14 landing site. The geological features of the area are significantly different from those of the VENERA-13 landing site. The planet surface at the site is a flat plain covered with a layer of fractured high-density (seemingly lithified) material that has clear boundaries of separate plates (Figure 3). The mechanical properties of the plates were studied by [9] in a

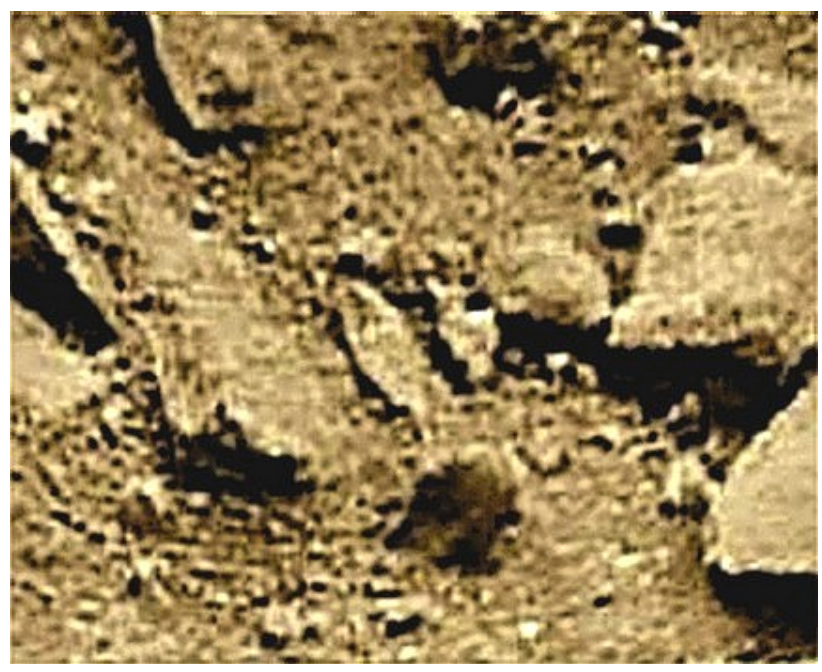

Figure 1. Panorama fragment obtained as a result of the VENERA-9 mission upon additional data processing. In the forefront, there is a small object seen from above, similar to a bear-cub. By its soft contours, the object is distinguished from the sharp stone edges. To the left beyond the object, over the ground, there propagate long furrows that terminate under the Bear-Cub. Apparently, these tracks indicate the preceding motion of the object. See [4] [5] for details. 


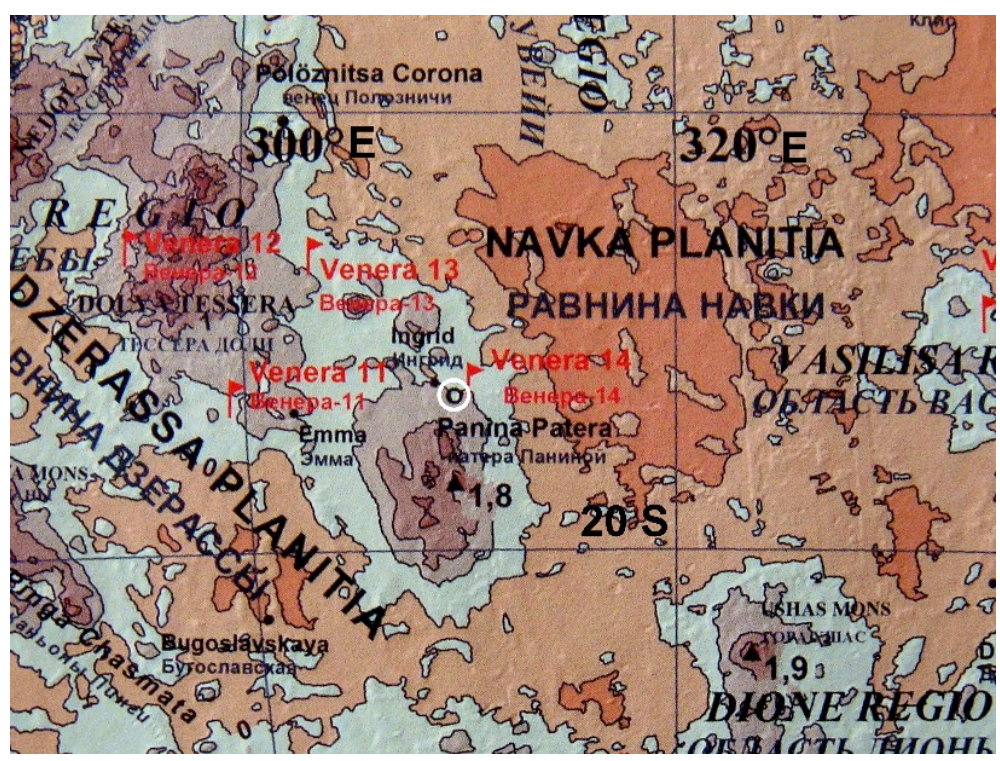

Figure 2. A fragment of the Venus surface map [8]. The white circle denotes the VENERA-14 landing site. At the Venus equator, $1^{\circ}$ of longitude/latitude equals $105.6 \mathrm{~km}$.

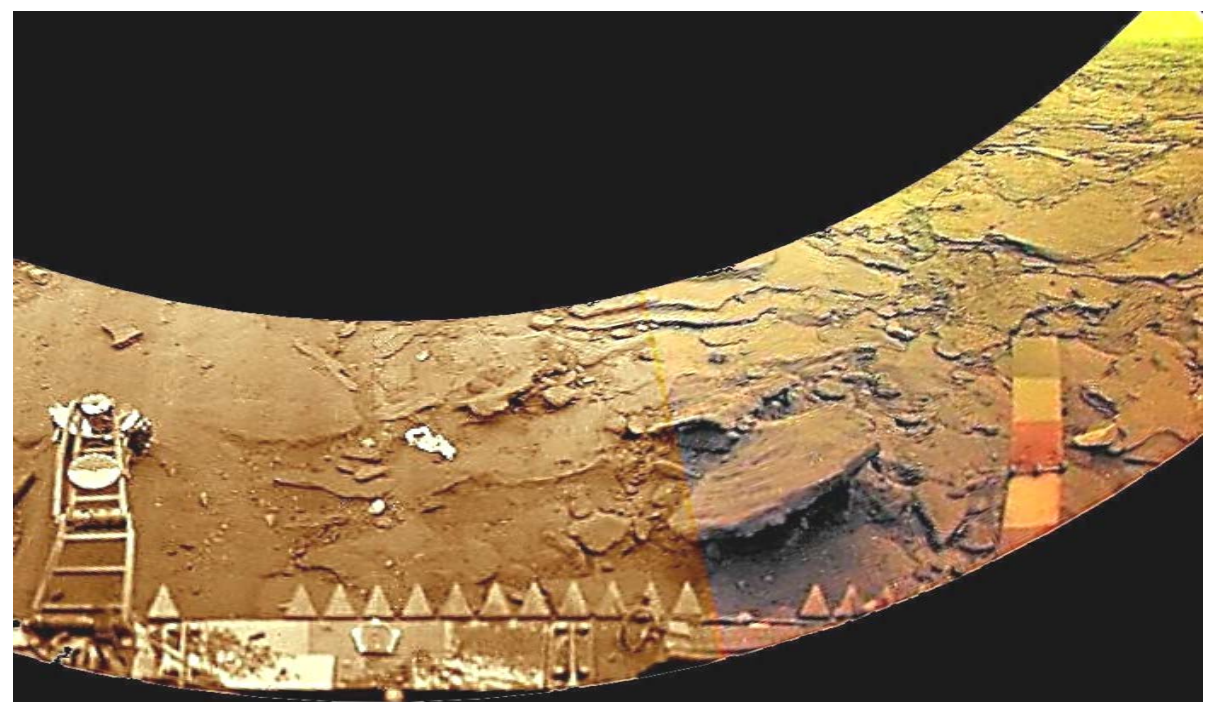

Figure 3. VENERA-14 panorama. The staircase truss (at left) is an unrolled lever system of the PrOP device that measures the load-bearing capacity of the ground. The unrolled truss is $60 \mathrm{~cm}$ long. In the right-hand part: color control strip.

special experiment. The material is of high load-bearing capacity that is 25 times greater, according to [9], than that of the loose ground at the VENERA-13 landing site. Its properties are roughly the same as those of basalt or granite.

\section{An Extraneous Body on the Surface of the Stratified Rocks}

A special issue of Kosmicheskiye Issledovaniya published in 1983, directly after the VENERA-13 and -14 missions, included a paper by K.P. Florensky et al. [7], that analyzed the area geology. "Strongly pronounced ... thin sub-horizontal stratification" was marked, with "large staircase shelves 1 - 2 to 5 - $10 \mathrm{~cm}$ high. ... The layers are different from each other in their reflective power." Then, the researchers focused on an unusual surface area that was close to the TV camera lens. They wrote, “Apart from the clearly stratified rocks, some of the pa- 
norama sections show rock outcrops without layering. For example, in the V14-1 panorama, to the right of the color test end, in a shallow depression of the microrelief, one can see a relatively dark, spotty, honeycomb surface with an odd zoned orientation of the spots. It appears that, from under the unconformable layers of the foliated unit, a rock surface of a different structure peeps out. This rock could underlie the foliated unit or make up some 'extraneous' entity within the foliated unit. At the left-hand boundary of the V14-2 panorama, there are some spotty shapes that might be outcrops of similar spotty rocks as well” [7] (1983, p. 345).

It remains to admire the insights of the K.P. Florensky team. In a recent publication [10], the author of this paper notes the other "spotty shapes in the V14-2 panorama" as possible Venusian flora objects, although it is rather difficult to underpin this hypothesis. At the same time, the "extraneous spotty, honeycomb entity at the V14-1 panorama" could have been the most amazing finding of K.P. Florensky et al. Repeatedly, they come back in their paper to the "small outcrops of spotty rocks". As is shown below, it only remains to regret that they failed to get further into research on that small "extraneous entity", which would have been quite worthwhile.

With respect to the assessment [9] of a rather high load-bearing capacity of the ground, the writers of the paper [7] comment that the capacity is greater than the estimate based on the dynamic parameters of the lander impact while landing, and they assume that the earlier assessment is wrong. If they are correct, then the error occurred for a reason that was difficult to foresee.

Similar to in all other VENERA landers, the front windows of the TV cameras were covered with caps that had to be blasted off after landing. The caps were removed, and one can see in Figure 3 that the PrOP-measuring cone was exactly on a cap (the white semi-cylinder $20 \mathrm{~cm}$ in size, see Figure 3). At the same time, the authors of [9] noted that the measuring cone proved to be beyond the cap; thus, the measured data were valid because they had to be related to the ground rather than to the cap.

\section{VENERA-14 TV-Panoramas That Contain “Extraneous Body” Images}

The objects found in the panoramic images from VENERA-14 draw upon the sequences of images that are organized in a way that is different from the earlier considered VENERA-13 panoramas [2]-[5]. The first sequence was designated Series 1 . Its data are almost entirely the same as in Series 6, which is evidenced by practically identical positions of the dot interferences. Series 9 repeat scanning was reproduced in Series 13 in accordance with the same schedule, but the data were different from Series 1 and 6 in both the positions of the interferences and the small shifts in the scene objects. First, full-scale $\left(180^{\circ}\right.$ each) black-and-white panoramas were scanned (within the 410 - $750 \mathrm{~nm}$ spectral range) by camera V-14-1 (designations as in [2] [3] [5], having 945 and 998 lines in 1/6 and 9/13 sequences, respectively. Afterward, only the following short image fragments were transmitted: red (590 - $720 \mathrm{~nm}$ ), green (490 - $610 \mathrm{~nm}$ ) and (useless) blue, of 330 - 336 lines each and, then, an incomplete $\left(118^{\circ}\right)$ black-and-white panorama BW-2. At the end of each sequence, there were full-scale BW-3 panoramas again, of 991 and 994 lines each. The time for one line of scanning was $780 \mathrm{~ms}$, and that of a complete panora- ma was approximately 13 min [1]. Considering the observed level of noise, the only red fragments that are ac- ceptable for analysis belong to the $1 / 6$ sequence. The green fragments can be treated in two series. The BW-2 panoramas do not contain the object under study. The noise level in panorama 1/6 BW-1 is small (Figure 3), while the 1/6 BW-3 panorama has noise all over. In the later panoramas (9/13 BW-1 and 9/13 BW-3), the noise level gradually rises, although the object under study, however strange it might be, remains almost noise-free (which was considered in [3]). Later, the 1/6 BW-3 panorama was substantially improved.

It is worthwhile to recall some data on the techniques of the experiment [1]. Two optomechanical cameras were installed on opposite sides of the lander. (This paper considers only the data that was received from camera 1 of VENERA-14.) The camera pupils were at $0.9 \mathrm{~m}$ above the land surface, and the upper boundary of the panoramas in the sector under study was approximately $3 \mathrm{~m}$ from the lander center. The axes of the scanning cameras were at $50^{\circ}$ to the vertical [1], which made it possible to resolve 2 millimeter details of the surface in immediate proximity to the lander and approximately $10 \mathrm{~m}$ at the mathematical horizon (at $3.3 \mathrm{~km}$ over the even surface). Illumination (with scattered sunlight) was 3 - $3.5 \mathrm{klx}$ [11], and the solar zenith angle during the experiment was $36^{\circ}$.

As has already been noted in [5], the landers operated much longer than was stated in [11]. The materials given below cover $1 \mathrm{hr} 33$ min of VENERA-14 operations on the planet surface. The data were referenced to time by using the transmitted line number contained in the housekeeping part of the experimental data. The angular resolution is $11^{\prime}$. Each line contains 211 image pixels and 41 elements of housekeeping data. To wait until 
the dust settles, a minute delay after landing was introduced before the cap was blasted off and transmission of the first panorama was commenced.

\section{Extraneous Honeycomb Entity}

The panorama fragments given below, similar to in the other papers of the series [2]-[6], have a limited resolution, only 11' per pixel, which is why one must be alert and patient while working with these images. The "extraneous honeycomb entity" (according to [7] terms) is in the rightmost part of Figure 3, to the right from the color control panel. It might be useful to mention that the panel is $10 \mathrm{~cm}$ wide, and each color field is square. The depression with the "extraneous honeycomb entity" inside is approximately $20 \mathrm{~cm}$ to the right from the panel. Figure 4 (frames 1 - 4) shows four untreated fragments of independent primary images of the object. Compared with the original images, the brightness and contrast of the images in Figure 4 have been leveled. The "extraneous entity" is in the center of each shot. The signal-to-noise ratio in the black-and-white panoramas is more than sufficient and gives a chance to use some of the primary images as they are. Their treatment is required only for further operations. Notwithstanding, the noise-to-signal ratios in the single-colored images are insufficient for a clear picture; as a result, these fragments were used exclusively for composite color image synthesis. Commonly, the stacking of repeat original images allows us to improve the identifiability of the details. However, an attempt to stack the pictures of the "extraneous honeycomb entity" revealed that, in a number of cases, depending on the choice of the stacking pilot point, when some parts of the pictures are aligned, some other parts become slightly out of focus.

The reason behind the alignment problem proved to be in a small shift in the object's position. Inasmuch as there are no less than four black-and-white images that are well suited for analysis even in their original quality (panoramas BW 1 and 3 of sequences 1/6 and 9/13) and no less than three same color-separated images, one can manage to produce a sequence of certain fragments as conventional "snapshots" of the same area (Figure 4).

During the operational period, due to the continuous rise of the temperature in the optic and electronic parts of the camera, the resolution varied: first, it became worse (shots 1-2, Figure 4); then, it improved (3); and then, it deteriorated again (4). The instants when the transmission of each fragment in Figure 4 commenced, starting from the beginning of the shot 1 transmission, were $2044 \mathrm{~s} \mathrm{(34} \mathrm{min} 04 \mathrm{~s}$ ), $3874 \mathrm{~s} \mathrm{(1} \mathrm{hr} 04 \mathrm{~min} 34 \mathrm{~s}$ ) and $5792 \mathrm{~s}$ (1 hr 36 min $32 \mathrm{~s}$ ). Comparing the transmission instants according to the line numbers, one can see that they do not completely match the table that is given in the express publication [1], while the content of the last panoramas was included in the table without any reference to time at all. The last line of the 9/13 BW-3 panorama,

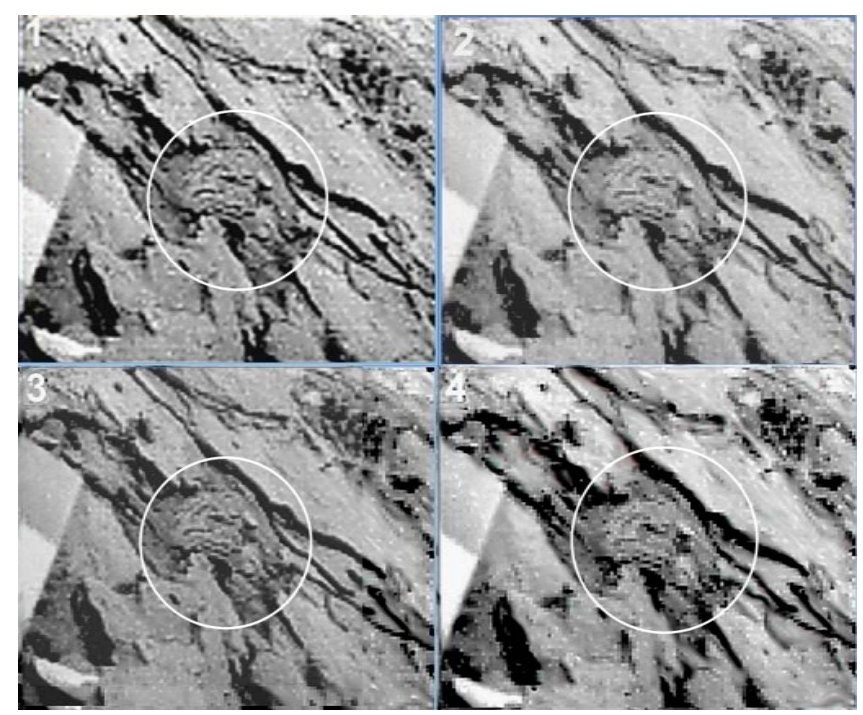

Figure 4. Consecutive shots of the "extraneous honeycomb entity" in the source black-and-white panoramas of VENERA-14 camera 1: 1 . file $B W-1$, sequence $1 / 6 ; 2$. file $B W-3$, sequence $1 / 6$; 3 . file BW-1, sequence $9 / 13$; 4 . file BW-3, sequence $9 / 13$. The shots are 34,30 and 32 min apart, respectively. 
from which shot 4 was taken, refers to the instant of $1 \mathrm{hr} 49 \min 27 \mathrm{c}$. Similar to in the case of VENERA-13, the cited data and the time references of the later series (Nos. 9 and 13 for VENERA-14) were revised further on [12].

The human vision capability of generalizing the details of unclear images is very diverse in different persons. In astronomy, there is a well-known classical case of dispute between Giovanni Schiaparelli, who managed to compile a map of Mars based on poorly visible patches, and Asaph Hall, who observed Mars visually and discovered two small Mars satellites. Asaph Hall absolutely could not agree with Schiaparelli's drawings. The image content of the "extraneous honeycomb entity" in Figures 4 and 5 was tentatively discussed by the author with his colleagues. Approximately $50 \%$ of them were able to see a coiled snake in the pictures, but the others were unable to notice it, even when it was distinguished with a circle around it as in Figure 5.

With due regard to the aforementioned problems in image stacking, the shots were prepared to be stacked allowed us to improve the image. Shot 4 of Figure 4 was not included in it. Figure 5 shows a treated image of the "extraneous honeycomb entity". Similar to in [2]-[6], no retouch was made whatsoever, except in the insert miniature at the bottom of Figure 5 for display purposes, where the "extraneous entity" contours are slightly highlighted. It is clear from Figure 5 that the regularly positioned spotty "cells" cover the object's surface. The object is in a small depression (5 - $10 \mathrm{~cm}$ deep), and it resembles a coiled snake. Moreover, it appears to possess certain terramorphic features. Conventional (provisional) names that have been suggested for the objects found on the Venus surface remain conventional; at the same time, the name "snake" is the most practical for the object under study precisely because of its terramorphic features.

\section{The Snake's Structure and Motion}

To estimate the snake's size, it is convenient to use the color control panel (seen in Figures 3 and 4). The height of each field is $10 \mathrm{~cm}$; however, at the distance where the snake is, the scale is $30 \%$ greater. The overall length of the snake amounts to approximately $40 \mathrm{~cm}$.

Apart from the snake, one can see some other unusual objects in Figures 5 and 6. In the 9-hour direction from the snake, on a flat bright stone, there is a dark object approximately $5-6 \mathrm{~cm}$ in size that was conventionally called a "dove". Its small dimensions do not permit us to identify any other details apart from the shadows under the body and under its protruding left part.

Because of the $50^{\circ}$ inclination of the scanning camera axis, the surface in Figure 4 appears to be sloping. The

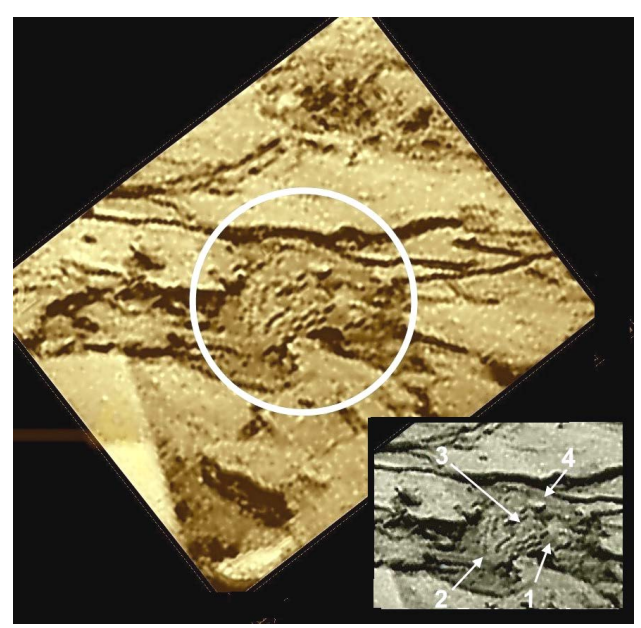

Figure 5. The image of "snake" made of stacked BW shots (except BW 3). For better viewing, the image is turned $38^{\circ}$ relative to Figure 4 . In the insert below, the arrows indicate the hypothetical structure of the snake as it can be perceived: $1-$ the head with an eye; 2 - the coiled body of the snake; 3-the crest; and 4 -the tail with protruding brighter detail. 


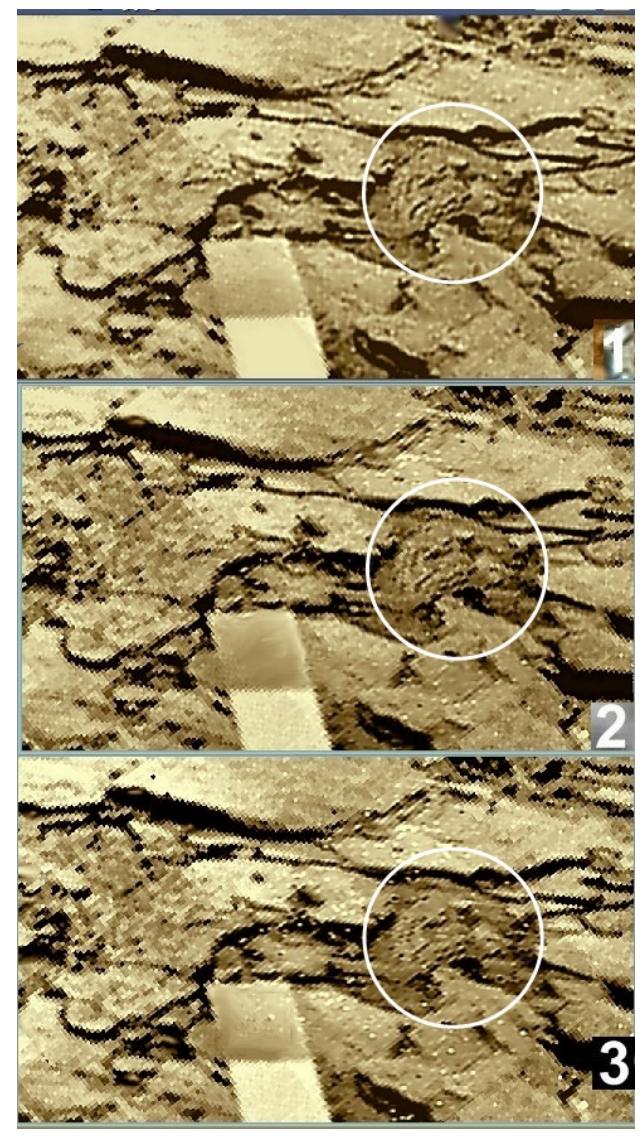

Figure 6. Treated images: (1) - a fragment from panorama BW-1, series 6; (2)—a fragment from panorama BW-1, series 9/13 and (3)-panorama BW-3, series 9/13. Comparing the fragments makes it possible to reveal some small relative moves of the snake's parts during the 1.5 hours.

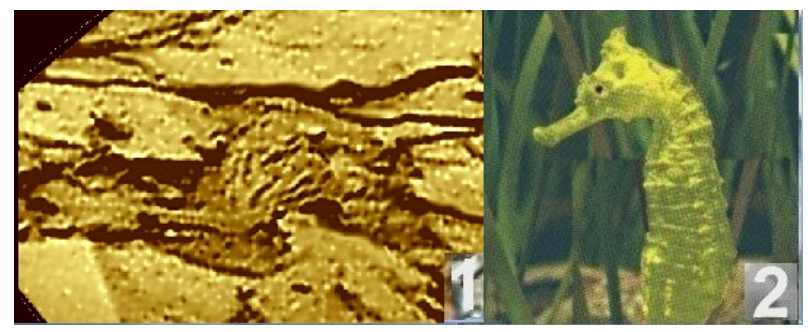

Figure 7. The snake's image (1) and the image of a sea horse (2) with a similar shape of the head. The actual sizes are quite different.

fragment was turned to improve the image display (Figures 5, 6 and 7), which made comparing the sizes more convenient. The geometric distortions in Figures 5, 6 have not been eliminated; however, the angle covering the picture is small $\left(34^{\circ}\right)$ and, as a result, the distortions are not essential.

Although a movement is insignificant, the snake's position gradually varies from shot to shot. The consecutive positions of the "extraneous entity" in the three sharpest panoramas are presented in Figure 6. Close examination of the shots allows us to see gradual, although very small, shifts in the relative positions of the snake's parts (the body, head and tail) and their positions relative to other parts of the snake as well as relative to the stone plates of the surface. It is more convenient to follow the shifts by using the Gif Animator software (a 
computer analogue of a blink comparator, an astronomical tool). It is possible that the "dove” shifts as well. However, it does not appear to be feasible to show in this paper the small shifts by blinking. As was noted in [13], whenever some shifts of the hypothetical Venus fauna are revealed, they are always extremely slow, no more than $1 \mathrm{~mm} / \mathrm{s}$. Comparing all of the shots of the sequence, one can arrive at the same or a lower rate for the relative shift of the snake's parts.

As has already been mentioned, the resolution in the images was limited by the angle of 11', which is why the identifiability is constrained even in the Figure 6 fragments that were treated by using "unsharp mask" processing. In the "green” pictures (not included here), the snake's body appears to be slightly brighter than in the black-and-white images. One can assume the snake’s structure as in Figures 5, 6, where the snake's hypothetic structure is presented and some parts of the "extraneous honeycomb entity" are indicated. The rightmost brighter part, the "head" (arrow 1, Figure 5), faces the camera with its round "eye" (the bright round spot can be noticed in any shot). The position of the spot leaves no doubt whatsoever as to its function. The "head" is approximately 6 - $8 \mathrm{~cm}$ in size and is reminiscent of the heads of many Earth beings (e.g., birds, reptiles and some marine animals). In Figure 7, the snake's head (1) is compared with the head of a sea horse (2), whose size is 2 3 times less. One can recall a long list of similar cases. The snake's head is topped with an indistinct bright mass that resembles a tuft that is found in numerous Earth species. To the left of the "eye”, there is a slightly elongated spot (Figure 5) that changes its shape in the successive shots of Figure 6. By analogy with Earth organisms, one can assume that it is a hearing organ (acoustic phenomena on Venus were noted in [4]). In Figures 5 and 6, above the body of the honeycomb entity (2), one can see a "crest" (3) that is approximately $4 \mathrm{~cm}$ long and is composed of separate elements that are bounded with an arc. In blinking, it was the crest that noticeably changed its position in the successive shots (shifting relative to a small dark area of the surface neighboring the "crest" on the left). The combined images can slightly deteriorate precisely because of their being stacked when the crest is chosen as the pilot detail for stacking (or, alternatively, the crest could become fuzzy in other stacking options). The head (1) and the bulging part (2) of the snake's body (Figure 5) also shift somewhat when blinking the successive shots shown in Figures 4 and 6. It appears that the snake ends with a long and broad "tail” (4) (see Figure 5), which butts on the right into a bright triangular rock fragment. As far as one can judge, the small bright detail that protrudes on top can be referred to as the "tail” as well because, in blinking, the detail successively shifts to the left along the tail. Left from the protruding detail, there are two small dark spots. A slight change in the tail position can also be traced by the relative change in the position of the two spots mentioned above. One cannot see any other spots on the tail. However, the shifts in the position of the snake's parts can hardly be noticed without blinking.

The fragments of the VENERA-14 panoramas that are under consideration are low noise, which offers a chance, in a number of cases, to arrive at distinct images even on the basis of a single panorama. The honeycomb surface of the snake's body with its regular spots stands out so strongly against the background of the surrounding stone plates that the entity cannot possibly be referred to as a geological object. Moreover, the snake can be included into the set of the most interesting findings of the Venus fauna because we succeeded in recording its successive motions. An interesting object called a "dove" is also notable, although one cannot identify the details of its structure. It is close to the snake, but that proximity could be accidental. However, Earth beings of a smaller size can be found in many cases to accompany larger ones and consume food wastes of the latter.

The established small shifts of the "snake" (and the "dove”) once again reveal that the style of physical activity of the hypothetical Venus fauna is very slow, which might be associated with some energy restrictions, as was suggested in [4] [5]. At the same time, a more credible reason for that observation might stem from the physical properties of the very material of which the Venus organisms are composed. As was noted repeatedly [3] [4] [10] [12], no liquid water can occur on the Venus surface; therefore, such organisms must rely on something else instead. Resins, for example, feature slow plasticity. An entity that is made of such a viscous material requires some noticeable time for any change in its shape. Can it be that the reason behind the tardiness of the Venus fauna is the properties of this viscous media?

The "snake" and the "dove" are not the only unusual objects in the VENERA-14 panoramas. Figure 8 shows an area that is close to the snake. Above the plate that is, in its turn, above the snake, there is a larger object of some periodic structure and, naturally, of unclear origin. The object rises above the surface, and its rather complicated structure could be difficult to associate with a geological formation. In the top part of Figure 8, at a left angle and in a round depression, one can see a bright object that has a regular symmetric radial structure that resembles another object that has a similar structure in the VENERA-13 panoramas, which is close to the "scorpion”. 


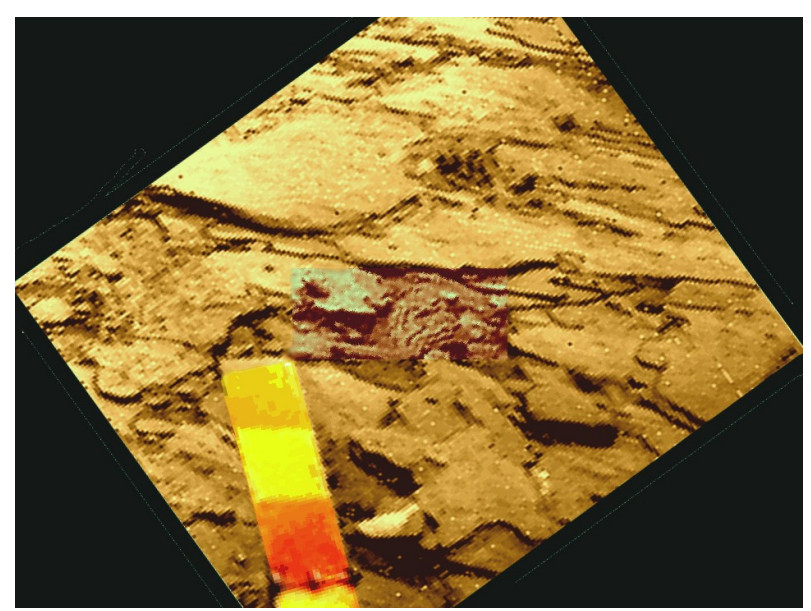

Figure 8. A color sector of the VENERA-14 panorama with the "snake" and other objects, demanding further research. Compared with the stone plates, the snake has a darker tone (which is not the case for the green tint pictures). The green tint of the plate with a "dove" on top could be a result of the processing.

\section{Conclusions}

The analysis of the VENERA-14 panoramas made it possible to reveal an object called a "snake" that is approximately $40 \mathrm{~cm}$ in length and possesses undeniable terramorphic features. The spotty, honeycomb surface of the snake's body is clearly different from the background, the surrounding stone plates. The "snake" can be referred to as one of the most interesting findings among the hypothetical Venus fauna. Another finding, a "dove", is of interest as well, although because of the distance and small size, the details of its structure can hardly be identified. The snake and the dove were found to move, although slowly, which confirms once again to the data of [2]-[6] [13] on the slow pattern of physical activity of the Venus fauna (as compared with the Earth fauna), which could be due to its energy constraints. However, there might be a different, no less plausible reason behind that, inasmuch as water, an active medium in Earth organisms, does not exist on Venus in its liquid form; therefore, there should be something else instead. Unlike water, the active medium that is the basis of the Venus fauna can feature slow plasticity. Resins, for example, possess such a property. This medium requires substantial time for any change in its shape. With respect to the terramorphic features of the Venus fauna [4]-[6] [12]-[14], in case they are confirmed, they would point at exceptionally important general laws of a living nature, yet undiscovered by science, occurring at absolutely different physical conditions, including the high-temperature, oxygen-free Venus environment or the settings of some types of exoplanets.

A number of VENERA-14 panoramas are low-noise, which gives a chance, as it is shown in the paper, to obtain clear images even on the basis of a single shot. On the whole, the VENERA-14 panoramas contain a sizeable number of uncommon objects that demand further research.

\section{References}

[1] Selivanov, A.S., Gektin, Yu.M., Gerasimov, M.A., et al. (1983) Continuation of the TV Study of the Surface of Venus by Means of Landers. Kosmicheskiye Issledovaniya, 21, 176-182.

[2] Ksanfomality, L.V. (2012) Possible Detection of Life on the Planet Venus, Doklady Physics, 57, 367-372. http://dx.doi.org/10.1134/S1028335812090029

[3] Ksanfomality, L.V. (2012) Venus as a Natural Laboratory for Search of Life in High Temperature Conditions: Events on the Planet on March 1, 1982. Solar System Research, 46, 41-53.

[4] Ksanfomality, L.V. (2012) Mobility and Other Features of Hypothetical Venusian Fauna. Doklady Physics, 57, 497501. http://dx.doi.org/10.1134/S1028335812120014

[5] Ksanfomality, L.V. (2013) Possible Signs of Life on the Planet Venus. International Journal of Astronomy and Astrophysics (IJAA), 3, 57-79. http://dx.doi.org/10.4236/ijaa.2013.31007 
[6] Ksanfomality, L.V. (2013) Hypothetical life detected on the planet Venus. International Letters of Chemistry, Physics and Astronomy (ILSPA), 10, 76-89.

[7] Florensky, K.P., Basilevsky, A.T., Kruchkov, V.P., et al. (1983) Geological-Geomorphological Analysis of the VENERA-13 and VENERA-14 Panoramas. Kosmicheskiye Issledovaniya, 21, 340-350.

[8] Lasarev, E.N., Odionova, R.J.F. and Shevchenko. V.V. (2012) Venus Relief Map. Moscow State University Press, Moscow.

[9] Kemurjian, A.L., Brodsky, P.N., Gromov V.V., et al. (1983) Preliminary Results of the Determination of Physics and Mechanics Properties of the Venus' Soil by the Soviet Automatic Probes VENERA-13 and VENERA-14. Kosmicheskiye Issledovaniya, 21, 323-330.

[10] Ksanfomality, L.V. (2013) The Hypothetic Flora of Venus. Doklady Physics, 58, 196-199. http://dx.doi.org/10.1134/S1028335813050029

[11] Moshkin, B.E., Economov, A.P., Moroz, V.I., et al., (1983) Spectrophotometric Experiment on VENERA-13, -14 Landers. Kosmicheskiye Issledovaniya, 21, 236-245.

[12] Ksanfomality, L.V. (2013) Snake-A New Object of Hypothetical Fauna of Venus. Doklady Physics, 58, 395-400.

[13] Ksanfomality, L.V. (2013) Looking for Mobility of Hypothetical Venusian Fauna. Advances in Zoology and Botany, 1, 25-29.

[14] Ksanfomality, L.V. (2013) An Object of Assumed Venusian Flora. Doklady Physics, 58, 204-206. 